\title{
Pathogenesis of Craniosynostosis
}

M.L. Walker, MD, FACS, FAAP, Division of Pediatric Neurosurgery, Primary Children's Medical Center, 100 North Medical Drive, Salt Lake City, UT 84113 (USA)

The article 'Pathogenesis of Craniosynostosis' by Becker and Hinton [1] adds further support to the concept that lambdoid synostosis is, indeed, a rare phenomenon. Only 3 of 41 sutures examined by them showed classical bony union across the suture. It is interesting that in all other forms of craniosynostosis, bony union is the standard by which craniosynostosis is judged. In the lambdoid, however, true bony union appears to be extremely rare. Since 1987 I have not seen a case that I thought was consistent with true bony fusion across the lambdoid sutures.

A more probable explanation is the pressure exerted by the child's position. Positional molding creates stress across the suture, and the inward prominence of the suture and the fibrous thickening are all consistent with the chronic pressure which these children exert on the posterior portion of their heads.

We know from clinical experience that the vast majority of these children will improve, either on their own or with treatment such as a helmet or banding. If true synostosis were present, the passage oftime and/or the use of helmet therapy would not benefit these patients. I congratulate the authors on providing these data to the medical literature. This is further evidence in support of the fact that the overwhelming majority of these children have positional molding and not true craniosynostosis.

Reference

Becker LE, Hinton DR: Pathogenesis of craniosynostosis. Pediatr Neurosurg 1995;22:1O4-107.

(C) 1995 S.KargerAG, Basel

$1016-2291 / 95 / 0225-0280$

$\$ 8.00 / 0$ 